\title{
A Highly Asymmetric Disc-Like Mesogen Based on the Tetrabenzotriazaporphyrin Macrocyclic Ring System
}

\author{
N. B. McKEOWN and C. C. LEZNOFF† \\ Department of Chemistry, York University, North York, (Toronto), Ontario, Canada M3J 1P3 \\ and
}

R. M. RICHARDSON and A. S. CHERODIAN

School of Chemistry, University of Bristol, Cantock's Close, Bristol, U.K. BS8 1TS

\begin{abstract}
Thermotropic mesomorphism has been observed in a highly substituted and asymmetrically shaped tetrabenzotriazaporphyrin (TBTAP) derivative. TBTAP has a ring system closely related to that of phthalocyanine, however the single meso-carbon provides an additional site for the attachment an alkyl chain. The TBTAP mesogen has a pentadecyl group at this site as well as a bulky neopentoxy substituent attached to each of the four peripheral benzo-portions of the macrocycle. Optical texture (focal-conic fan) and X-ray diffraction studies suggest that the TBTAP has a mesophase with a lamellar structure analogous to that of the smectic A phase of rod-like molecules. Such a mesophase has not been observed previously from discotic compounds.
\end{abstract}

Keywords: thermotropic mesomorphism, tetrabenzotriazaporphyrins, smectic, discotic

\section{INTRODUCTION}

Phthalocyanines and porphyrins have enormous potential as molecular semiconductors. ${ }^{1}$ In addition, suitably substituted derivatives of these macrocycles also form discotic liquid crystals and hence, there is a real possibility of fabricating electronic devices based on self assembled organic semiconductors. ${ }^{2}$ To this end, many symmetrical octaalkyl substituted derivative of porphyrin, ${ }^{3,4}$ tetraazaporphyrin, ${ }^{5}$ phthalocyanine, ${ }^{6-15}$ (tetrabenzotetrazaporphyrin) and tetrapyrazinotetraazaporphyrin, ${ }^{16}$ have been prepared and their mesophases investigated.

Asymmetrical derivatives of phthalocyanines are much more difficult to prepare, ${ }^{17-21}$ nevertheless a phthalocyanine mesogen in which nitrile groups replaced two of the long alkyl chains has been reported. ${ }^{22}$ Octaalkyl porphyrin derivatives

$\dagger$ Author to whom correspondence should be addressed. 
with a polar group (e.g., nitrile) at one of the meso positions, have also been prepared and show mesophase behaviour. ${ }^{4}$ In both cases the asymmetrical substitution of the macrocycle had little effect on the structure of the resultant mesophase, when compared with those of the analogous symmetrical compounds.

The tetrabenzotriazaporphyrin (TBTAP) ring system differs from that of phthalocyanine only by a methine group, instead of a nitrogen, at a meso position (see Figure 1). The unsubstituted TBTAP (1) has been made from a reaction between phthalonitrile and methylmagnesium iodide. ${ }^{23}$ Recently, we have shown that substitution of TBTAP at the meso-carbon is possible and isolation of the product facile if longer alkyl Grignard reagents are reacted with phthalonitrile. In addition, highly substituted TBTAPs were prepared by using alkyl derivatives of phthalonitrile as precursors. For example, 2,9,16,23-tetrakis(2,2-dimethylproproxy)-27pentadecyl-29H,31H-tetrabenzo $[b, g, l, q][5,10,15]$ triazaporphine (2) and 2,9,16,23tetra - kis $(2,2$ - dimethylethyl) - 27 - pentadecyl - $29 \mathrm{H}, 31 \mathrm{H}$ - tetrabenzo $[b, g, l, q]$ $[5,10,15]$ triazaporphine $(3)$ were synthesized by reacting hexadecylmagnesium chloride with 4-neopentoxyphthalonitrile and 4-tert-butylphthalonitrile, respectively ${ }^{24}$ (Figure 1). The presence of the long alkyl chain group facilitated the separation of the TBTAPs, by chromatographic methods, from the phthalocyanine by-products.

This paper describes the preliminary results from the investigation into the interesting thermotropic mesomorphic behaviour of TBTAP 2 unlike that of TBTAP 3 which forms no liquid crystal phases.

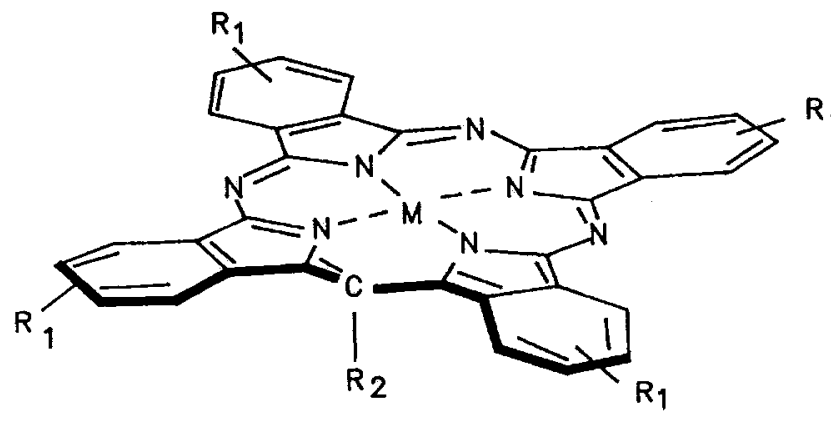
$1 \quad R_{1}=H$,
$R_{2}=H_{1}$
$M=H_{2}$
$2 \mathrm{R}_{1}=\mathrm{OCH}_{2} \mathrm{C}\left(\mathrm{CH}_{3}\right)_{3}$
$\mathrm{R}_{2}=\left(\mathrm{CH}_{2}\right)_{14} \mathrm{CH}_{3}$
$M=H_{2}$
$3 \quad \mathrm{R}_{1}=\mathrm{C}\left(\mathrm{CH}_{3}\right)_{3}$
$\mathrm{R}_{2}=\left(\mathrm{CH}_{2}\right)_{14} \mathrm{CH}_{3} \quad \mathrm{M}=\mathrm{H}_{2}$

FIGURE 1 The tetrabenzotriazaporphyrin ring system. 


\section{EXPERIMENTAL}

The TBTAPs 2 and 3 were synthesised as described previously. ${ }^{24}$ The purity of both compounds are confirmed using elemental microanalysis and thin layer chromatography. Further characterisation of compounds were established using Fast Atom Bombardment (FAB) mass spectrometry in addition to UV/Visible, IR and ${ }^{1}$ H N.M.R. spectroscopy. Samples of TBTAP were heated between two untreated glass slides on a hot stage and optical textures were observed by using a Leitz polarising microscope. Calorimetric measurements were performed using a PerkinElmer DCS-2C differential scanning calorimeter. The powder X-ray diffraction patterns from the solid and mesomorphic phases of TBTAP 2 were achieved using a 2-D position sensitive detector with copper $\mathrm{K} \alpha$ radiation. The sample was contained in a sealed Lindemann tube for these experiments. Molecular dimensions for TBTAP 2 were estimated from CPK molecular models.

\section{RESULTS}

Using polarized optical microscopy it was observed that TBTAP 3 melts from a solid into a fluid isotropic liquid at $119^{\circ} \mathrm{C}$. In contrast, TBTAP 2 was a highly viscous birefringent liquid at $130^{\circ} \mathrm{C}$. The phase change from solid to mesophase occurred over a broad temperature range $\left(100-125^{\circ} \mathrm{C}\right)$. The appearance of the mesophase (Figure 2) did not alter when the temperature was raised to $300^{\circ} \mathrm{C}$ (the upper limit of the hot stage used in this study), although the liquid became less viscous at higher temperatures. When cooled below $100^{\circ} \mathrm{C}$ the compound solidified, but retained the birefringent texture of the mesophase. The standard technique of viewing the natural (spontaneous) texture of the mesophase formed by a gradual cooling of the isotropic liquid could not be applied to this material due to its high clearing point. However, the TBTAPs proved to be stable to thermal decomposition

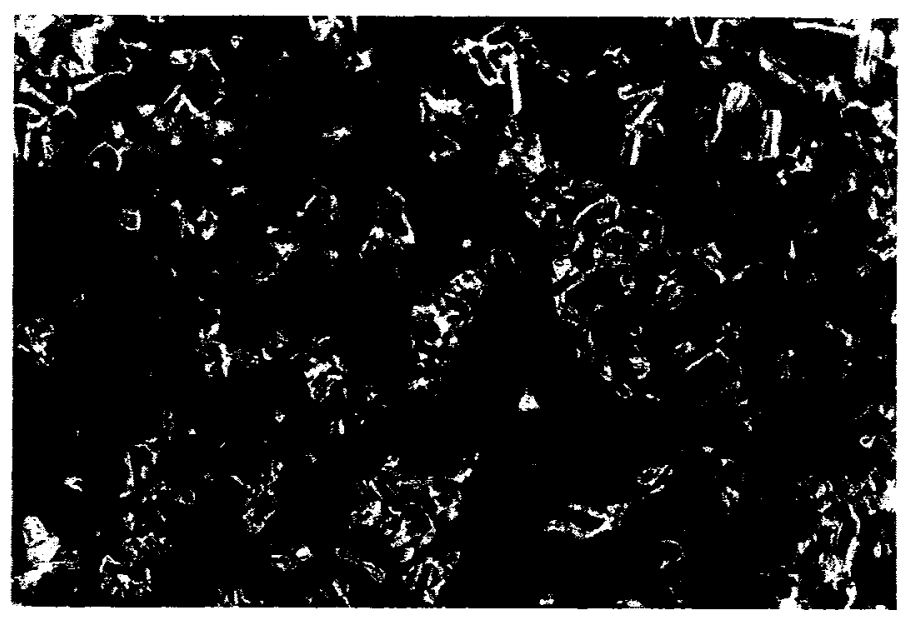

FIGURE 2 The optical texture of TBTAP 2 obtained from heating the amorphous solid $\left(160^{\circ} \mathrm{C}\right.$, crossed Polarisers). 
even at elevated temperatures as no color change was observed. Hence, it was possible to achieve the fluid isotropic phase by briefly heating the sample between two untreated glass slides, over a naked flame. Following rapid cooling to room temperature, the solidified sample appeared mainly homeotropic when viewed under a polarising microscope but focal-conic fan textural areas were also apparent (Figure 3). The appearance of this sample remained unchanged when reheated to produce the mesophase, using the hot stage. Therefore, we believe that the textures represented by Figures 2 and 3 both belong to the same mesophase of TBTAP 2 but that the texture seen on heating the sample above the solid to mesophase transition is masked by that of the amorphous solid phase.

A Differential Scanning Caliometric (DSC) scan (heating rate $=10^{\circ} \mathrm{C} \mathrm{min}-1$ ) of TBTAP 2 proves to be rather featureless with only a few small peaks $(\Delta \mathrm{H}<$ $0.1 \mathrm{kcal} / \mathrm{mole}$ ) discernible between $100^{\circ} \mathrm{C}$ and $125^{\circ} \mathrm{C}$. No other phase change was apparent on heating the sample to $370^{\circ} \mathrm{C}$. Therefore, the clearing point of TBTAP 2 must occur at a higher temperature than $370^{\circ} \mathrm{C}$.

The X-ray diffraction patterns acquired from the solid phase (at $24^{\circ} \mathrm{C}$ ) and nonoriented mesophase (at $159^{\circ} \mathrm{C}$ ) of compound 2 are very similar (Figure 4). An intense peak can be observed in the small angle region for both the solid and the metaphase with Bragg spacings (d) $20.9 \AA$, respectively. In addition, two very diffuse halos located at positions corresponding to $d$ spacings of around $5.0 \AA$ and $3.7 \AA$ are also present.

\section{DiSCUSSION}

The peripherally substituted TBTAPs 2 and 3 were prepared as ten different regioisomers and as such do not form crystalline solids. The absence of a sharp phase transition from solid to a mesophase for TBTAP 2 reflects the fact that 2 is a mixture of isomers. In addition, the X-ray diffraction analysis of the solid and

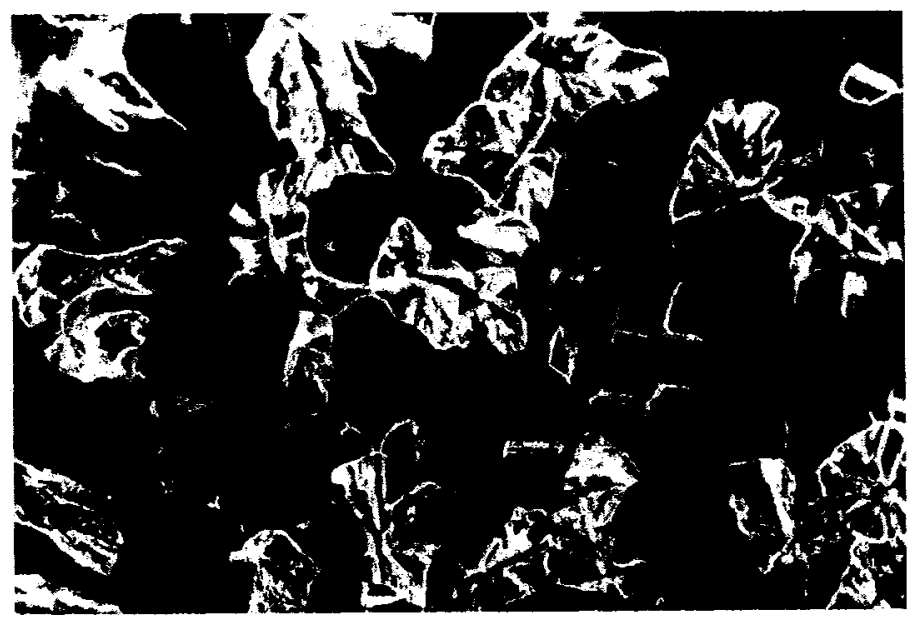

FIGURE 3 The optical texture of TBTAP 2 obtained from rapid cooling from the isotropic phase $\left(25^{\circ} \mathrm{C}\right.$, crossed polarisers). 


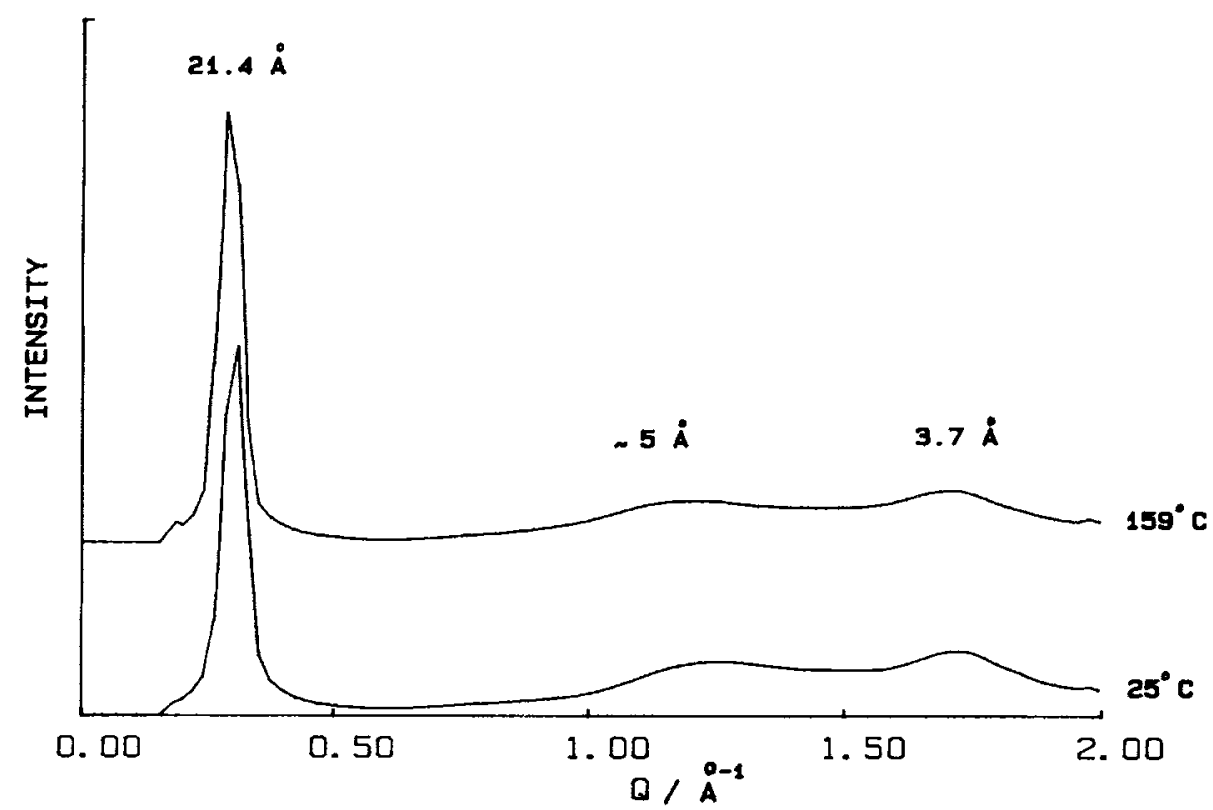

FIGURE 4 The X-ray diffraction patterns obtained from the amorphous solid $\left(25^{\circ} \mathrm{C}\right)$ and the mesophase $\left(159^{\circ} \mathrm{C}\right)$.

mesophase of TBTAP 2 (Figure 4) indicates that the molecular arrangement within both of these phases is similar. This similarity is consistent with retention of the mesophase texture when TBTAP 2 was cooled to the solid phase and the very small enthalpy change associated with the solid to mesophase transition. The broad temperature range over which the mesophase occurs $\left(>250^{\circ} \mathrm{C}\right)$ is typical for phthalocyanine-like mesogens. ${ }^{6-15}$

Disc-like mesomorphic compounds (discogens) such as the phthalocyanines tend to form mesophases in which the molecules are cofacially stacked in columns. These columns arrange in a 2-dimensional lattice with either hexagonal ${ }^{6-13}$ or rectangular symmetry. ${ }^{10,11} \mathrm{~A}$ hexagonal array, in which there is no long range translational ordering of the molecules along the axis of the column, has been designated as disordered hexagonal $\left(D_{h d}\right)$ by Destrade et al..$^{25}$ The 'disorder' refers to the relative positions of the molecules within the columns and not the arrangement of the columns themselves. This type of mesophase is associated with focal-cone fan textures similar to the one seen on cooling 2 from the isotropic phase (Figure 3 ) and is commonly encountered in studies of mesogens based on the phthalocyanine structure..$^{7-11,13}$ Indeed, the asymmetrically substituted phthalocyanine derivative reported by Piechocki and Simon, form a $D_{h d}$ mesophase with lattice parameters consistent with a staggared arrangement of the molecules within the columns. ${ }^{22}$ This phthalocyanine derivative gave a diffraction pattern almost identical to those obtained from the analogous symmetrical compounds, indicating that the columns are forming similarly ordered arrays.

The existence of a regular $D_{h d}$ mesophase, with the TBTAP molecules randomly positioned with respect to rotation around the axis of the column, would be in- 
dicated by at least three sharp Bragg reflections in the small-angle $\mathrm{X}$-ray diffraction pattern. The reciprocal spacings of these reflections would be in the ratio of $1: \sqrt{ } 3: \sqrt{ } 4$. Clearly, the powder X-ray diffraction pattern obtained from the mesophase of 2 is not consistent with such an ordered 2-dimensional hexagonal symmetry. It is possible that a less ordered columnar structure might only show a first order Bragg reflection, as reported recently for a metacyclophane mesogen. ${ }^{26}$ Assuming that the intense peak corresponding to a distance of $21.4 \AA$ is associated with the first order peak from a hexagonal lattice, the centre-to-centre intercolumnar distance would only be $24.1 \AA$. A column in which the TBTAP molecules are randomly displaced, with respect to the axis of the column, would be expected to have a diameter significantly greater than that of the tetraneopentoxy-substituted macrocycle (diameter $24 \AA$ ) to accommodate the long pentadecyl chain. Hence, it is doubtful that the mesophase of TBTAP 2 can be classified as $D_{h d}$ in spite of the optical texture it displays.

Discotic mesophases in which there is a rectangular packing of the columns $\left(D_{r d}\right)$ have been reported for phthalocyanine derivatives. ${ }^{10,11}$ However, the textures and $\mathrm{X}$-ray diffraction patterns associated with these mesophases were not those we observed from the mesophase of TBTAP 2.

Recently, a discotic nematic $\left(N_{d}\right)$ mesophase has been reported from a phthalocyanine derivative with eight branched alkyl side chains. ${ }^{15}$ Similarly, the sterically large neopentyl substituents would be expected to discourage the formation of a columnar structure within the mesophase of TBTAP 2. ${ }^{27,28}$ Although the X-ray diffraction pattern from TBTAP 2 show quite a disordered molecular arrangement, the focal-conic texture and high viscosity of the mesophase strongly indicates that it is not of a $N_{d}$ type. Hence, it is apparent that the mesophase formed by the TBTAP 2 cannot be easily classified as one of the known arrangements formed by phthalocyanine mesogens.

Smectic-like mesophases have been obtained from a homologous series of disclike bis(1,3-di(p-n-alkoxyphenyl)-propane-1,3-dianato)copper(II) complexes ${ }^{29,30}$ These discogens are similar to TBTAP 2 in that their shape is not highly symmetrical. For example, the dodecyloxy derivatives is reported to be around $40 \AA$ long and only $33 \AA$ across. The mesophase produced by these compounds has been designated discotic lamellar $\left(D_{L}\right)$ and is characterised by a tilting of the molecules within the layers, similar to that found in the smectic $\mathrm{C}$ phase of rod-like mesogens. The lamellar arrangement is apparent from the spacing of the reflections in the $\mathrm{X}$ ray diffraction pattern $(1: 1 / 2: 1 / 3)$. The higher order lamellar reflections are an indication of the regular nature of this $D_{L}$ mesophase.

Many smectic phases derived from rod-like molecules exhibit a focal-conic fan texture such as the one displayed by TBTAP 2 . Generally though, only the relatively disordered smectic A phase $\left(S_{A}\right)$ display this texture spontaneously on forming from the isotropic phase. ${ }^{31,32}$ The powder X-ray diffraction pattern from a $S_{A}$ phase gives an inner ring associated with the lamellar spacing and a diffuse outer halo related to the liquid-like arrangement of molecules within the layers, often only the first order lamellar rings are observed. ${ }^{32,33}$ If TBTAP 2 forms a discotic lamellar mesophase which is analogous to the smectic A phase formed by rod-like molecules, then the lamellar spacing would correspond to the location of the intense diffraction 
peak at $21.4 \AA$. This value is close to the diameter of the aromatic macrocycle portion of the molecule ( $24 \AA$ ) and would imply that the molecules are forming layers in which their long alkyl chains are roughly parallel to the Smectic plane. ${ }^{7}$

Rod-like molecules arranged in the smectic $\mathrm{A}$ phase are considered to rotate freely about their long molecular axes and therefore, sweep out a cylindrical shaped volume with a diameter in the order of $5 \AA .{ }^{33}$ This gives the characteristic diffuse ring in the wide angle region of the X-ray diffraction pattern. In the case of TBTAP 2 , rotation would be hindered due to their disc-like molecular shape and this would lead to the plane of the molecules being on average parallel. This arrangement within the smectic layer might be expected therefore, to give rise to a weak halo related to the average molecular spacing parallel to the plane of the macrocyclic ring. Previously, the intermolecular distance has been measured at around $3.5 \AA$, in phthalocyanine mesophase $\mathrm{s}^{8,9,15}$ and the broad halo at $3.7 \AA$, obtained in the wide angle diffraction pattern of the mesophase of TBTAP 2 , is in reasonable agreement with this value. The other diffuse halo, located at around $5.0 \AA$, is typical of discotic phases and is related to the presence of long alkyl chains. ${ }^{6-8,12,15,27}$

The X-ray diffraction pattern and optical texture of the mesophase of TBTAP 2 are both consistent with a lamellar arrangement of the molecules in which only a nematic-like ordering of the molecules is present with the smectic plane. Work is in progress to obtain aligned samples of the mesophase of TBTAP 2 to confirm, by X-ray diffraction, the postulated lamellar structure.

\section{CONCLUSION}

We believe that the mesophase of TBTAP 2 has a lamellar structure analogous to the molecular arrangement found in the Smectic A phase of rod-like mesogens. This relatively disordered structure, compared with the columnar mesophases obtained from phthalocyanine derivatives substituted with only straight alkyl chains, is most probably caused by the bulky neopentyl side chain. The lamellar array would also be a space efficient packing arrangement for the accommodation of the long pentadecyl chains. It is of interest that TBTAP 2 forms this highly stable mesophase whereas TBTAP 3 exhibits no mesophase behaviour. This may be due to the greater flexibility of the neopentyl group compared with that of the tertbutyl substituent.

It is easy to anticipate that the versatility offered by the simple one-reaction synthesis of the TBTAPs may facilitate the preparation of many discogens based on this macrocycle. It would be particularly interesting to prepare intrinsically asymmetric phthalocyanine analogs containing two different types of side chains. These analogs would help, in the future, to extend the range of properties and potential applications of semi-conducting organic mesogens.

\section{Acknowledgment}

Financial support by the Natural Sciences and Engineering Research Council of Canada is gratefully acknowledged. 


\section{References}

1. J. Simon and J. J. Andre, Molecular Semiconductors (Springer, Berlin, 1985).

2. J. Simon and C. Sirlin, Pure and Appl. Chem., 61, 1625 (1989).

3. B. A. Gregg, M. A. Fox and A. J. Bard, J. Chem. Soc, Chem. Commun., 1134 (1987).

4. B. A. Gregg, M. A. Fox and A. J. Bard, J. Am. Chem. Soc., 111, 3024 (1989).

5. P. Doppelt and S. Huille, New J. Chem., 14, 607 (1990).

6. C. Piechocki, J. Simon, A. Skoulios, D. Guillon and P. Weber, J. Am. Chem. Soc., 104, S245 (1982).

7. D. Guillon, A. Skoulios, C. Piechocki, J. Simon and P. Weber, Mol. Cryst. Liq. Cryst., 100, 275 (1983).

8. D. Guillon, P. Weber, A. Skoulios, C. Piechocki and J. Simon, Mol. Cryst. Liq. Cryst., 130, 223 (1985).

9. M. J. Cook, M. F. Daniel, K. J. Harrison, N. B. McKeown and A. J. Thomson, J. Chem. Soc., Chem. Comm., 1086 (1987).

10. M. J. Cook, N. B. McKeown, A. J. Thomson, K. J. Harrison, R. M. Richardson, A. N. Davies and S. J. Roser, Chemistry of Materials, 1, 387 (1989).

11. A. S. Cherodian, A. N. Davies, R. M. Richardson, M. J. Cook, N. B. McKeown, A. J. Thomson, J. Feijoo, G. Ungar and K. J. Harrison, Mol. Cryst. Liq. Cryst., in press.

12. D. Masurel, C. Sirlin and J. Simon, New J. Chem., 11, 455 (1987).

13. K. Ohta, L. Jacquemin, C. Sirlin, L. Bosio and J. Simon, New J. Chem., 12, (1988).

14. I. Cho and Y. Lim, Mol. Cryst. Liq. Cryst., 154, 9 (1988).

15. D. Lelievre, M. A. Petit and J. Simon, Liq. Cryst., 4, 707 (1989).

16. K. Ohta, T. Watanabe, T. Fujimoto and I. Yamamoto, J. Chem. Soc., Chem. Comm., 1611 (1989).

17. C. C. Leznoff, Phthalocyanines - Properties and Applications, ed. by C. C. Leznoff and A. B. P. Lever (VCH, New York, 1989), Chapter 1.

18. T. W. Hall, S. Greenberg, C. R. McArthur, B. Khouw and C. C. Leznoff, Nouv. J. Chem., 6, 653 (1982).

19. C. C. Leznoff, S. Greenberg, B. Khouw and A. B. P. Lever, Can. J. Chem., 65, 1705 (1987).

20. C. C. Leznoff and S. Greenberg, Tetrahedron Lett., 30, 5555 (1989).

21. N. B. Mckeown, I. Chambrier and M. J. Cook, J. Chem. Soc., Perkin Trans. 1, 1169 (1990).

22. C. Piechocki and J. Simon, J. Chem. Soc., Chem. Comm., 259 (1985).

23. P. A. Barrett, R. P. Linstead, G. A. P. Tuey and J. M. Robertson, J. Chem. Soc., 1809 (1939).

24. C. C. Leznoff and N. B. McKeown, J. Org. Chem., 55, 2186 (1990).

25. C. Destrade, P. Foucher, H. Gasparoux, N. H. Tihn, A. M. Levelut, J. Mathete, Mol. Cryst. Liq. Cryst., 106, 121 (1984).

26. G. Cometti, E. Dalcanale, A Du Vosel and A-M. Levelut, J. Chem. Soc., Chem. Comm., 163 (1990).

27. H. Gasparoux, Mol. Cryst. Liq. Cryst., 63, 231 (1981).

28. N. H. Tinh, H. Gasparoux and C. Destrade, Mol. Cryst. Liq. Cryst., 68, 101 (1981).

29. K. Ohta, H. Muroki, A. Takagi, K-I. Hatada, H. Ema, I. Yamamoto and K. Matsuzaki, Mol. Cryst. Liq. Cryst., 140, 131 (1986).

30. H. Sakashita, A. Nichitani, Y. Sumiya, H. Terauchi, K. Ohta and I. Yamamoto, Mol. Cryst. Liq. Cryst., 163, 211 (1988).

31. D. Demus and L. Richter, Textures of Liquid Crystals, (Verlag Chemie Weinheim, New York, 1978).

32. G. W. Gray and J. W. G. Goodby, Smectic Liquid Crystals - Textures and Structures, (Leonard Hill, Glasgow, 1984).

33. A. De Vries, Mol. Cryst. Liq. Cryst., 131, 125 (1985). 